\title{
Auditory temporal cues can modulate visual representational momentum
}

\author{
Wataru Teramoto, Souta Hidaka, Jiro Gyoba, and Yôiti Suzuki \\ Tohoku University, Sendai, Japan
}

\begin{abstract}
In representational momentum (RM), the final position of a moving target is mislocalized in the direction of motion. Here, the effect of a concurrent sound on visual RM was demonstrated. A visual stimulus moved horizontally and disappeared at unpredictable positions. A complex tone without any motion cues was presented continuously from the beginning of the visual motion. As compared with a silent condition, the RM magnitude increased when the sound lasted longer than and decreased when it did not last as long as the visual motion. However, the RM was unchanged when a brief complex tone was presented before or after the target disappeared (Experiment 2) or when the onset of the long-lasting sound was not synchronized with that of the visual motion (Experiments 3 and 4). These findings suggest that visual motion representation can be modulated by a sound if the visual motion information is firmly associated with the auditory information.
\end{abstract}

Localizing a moving object is an essential ability required to avoid obstacles and to take other appropriate actions on them. It appears that in everyday life, humans carry out such tasks without any difficulty. However, in laboratory experiments, several types of mislocalizations of a moving object have been reported (Freyd \& Finke, 1984; Frölich, 1923; Nijhawan, 1994; Thornton, 2002). One such example is that of representational momentum (RM), in which the final position of a moving visual stimulus is mislocalized forward in the direction of motion (see Hubbard, 1995b, 2005, for reviews). Freyd and Finke (1984) termed this mislocalization "representational momentum" because motion of a moving stimulus is prolonged in the direction of the object's implied path of motion, and this is similar to the manner in which motion of a physical object is prolonged. This is an analogy to the physical momentum of real-world objects. A number of variables influence the magnitude of visual RM, such as velocity (Freyd \& Finke, 1985; Hubbard \& Bharucha, 1988), acceleration (Finke, Freyd, \& Shyi, 1986), direction of motion (Halpern \& Kelly, 1993), shape (Nagai \& Yagi, 2001), expectation (Hubbard \& Bharucha, 1988), and conceptual context (Reed \& Vinson, 1996; Vinson \& Reed, 2002). Although the mechanisms underlying RM remain unclear, it is agreed that some extrapolation processes in the visual system may be involved in order to maintain a mental representation of a moving visual stimulus for a brief period after the visual stimulus physically vanishes.

Although the phenomenon has been investigated particularly in the visual modality, a similar effect was reported in the auditory modality as well. When listeners were asked to judge the final pitch of a series of ascending or descending pitches, the final pitch was heard as having shifted in the upward or downward direction of implied change (Hubbard, 1995a; Johnston \& Jones, 2006; Kelly \& Freyd, 1987). This auditory RM was influenced by the velocity and acceleration of the frequency changes of sounds in a manner similar to the visual RM (Freyd, Kelly, \& DeKay, 1990). Getzmann, Lewald, and Guski (2004) investigated auditory RM in the spatial domain, showing that the final position of the auditory target was displaced in the forward direction of auditory motion. They also showed that an increase in the velocity of the auditory motion caused no increase in the magnitude of auditory RM in the spatial domain. This finding suggests that the underlying mechanism for auditory RM in the spatial domain may be different from that for visual RM. Although there were some inconsistencies between the visual and auditory RMs, these studies suggest that forward displacements of the final position of a moving object in the direction of motion reflect one of the fundamental properties across sensory modalities.

In everyday life, humans perceive a real object by hearing as well as seeing. Recent research on multimodal interaction has shown that different sensory modalities are more closely related and mutually interactive than what classical views had assumed. This is true for audiovisual processing, as illustrated in the spatial ventriloquism effect (Bertelson \& Aschersleben, 1998; Radeau, 1994), dynamic visual capture (see Soto-Faraco, Kingstone, \& Spence, 2003, for a review), auditory driving (Gebhard \& Mowbray, 1959; Shipley, 1964; Welch, DuttonHurt, \& Warren, 1986), the temporal ventriloquism effect (MoreinZamir, Soto-Faraco, \& Kingstone, 2003; Vroomen \& de Gelder, 2004), and so on. Although RM phenomena have 
continued to be of interest, little is known about multimodal interactions on RM phenomena. Recently, Hubbard and Courtney (2010) investigated the effect of linear frequency change of sounds (ascending or descending frequency) on visual RM and the effect of visual information on auditory RM induced by the linear frequency change. They reported the enhancement of the auditory RM when metaphorically congruent visual motion - ascending frequency with upward motion and descending frequency with downward motion - was presented. With regard to the effect of audition on visual RM, they reported that the changing frequency had little effect on visual RM when vertical visual motion was presented but was effective for visual representational gravity-mislocalization of the final position of a moving visual stimulus in the direction of implied gravity (Hubbard, 1990, 1995b, 1997; Hubbard \& Bharucha, 1988) - when horizontal visual motion was presented. Thus far, it has been reported that the auditory system is usually superior to the visual system in temporal processing and that the visual system is usually superior to the auditory system in spatial processing (Welch \& Warren, 1980, 1986; but see also Handel, 1988; Kubovy, 1988). This conceptual framework could account for most of the observed influences of auditory information on visual motion perception and the lack of the metaphorical congruency effect of sounds on visual RM in Hubbard and Courtney. Therefore, the present study focused on the effect of the temporal aspect of auditory information on visual RM.

Two recent studies that have shown the effect of auditory information on localizations of a moving visual stimulus are informative for this purpose (Heron, Whitaker, \& McGraw, 2004; Vroomen \& de Gelder, 2004). Heron et al. demonstrated that the bounce position of a horizontally moving visual object against a vertical virtual surface appeared to be displaced depending on the timing at which a brief sound was delivered (and its temporal certainty) and on the spatial certainty of the visual stimulus. As compared with a no-sound condition, when the sound occurred prior to the actual bounce, the bounce position appeared to be displaced in the direction opposite to the preceding visual motion (i.e., before the actual position of the bounce). On the other hand, when the sound occurred after the actual bounce, the bounce position appeared to be displaced in the forward direction of the preceding visual motion (i.e., after the actual position of the bounce). It should be noted that these phenomena could be observed only when visual positional uncertainty was high and auditory temporal uncertainty was low.

Another example was reported using flash-lag displays, wherein a flash is perceived to be misaligned with a moving visual object presented near it, even if the flash and object are physically presented at the same location (Nijhawan, 1994). Vroomen and de Gelder (2004) demonstrated that the magnitude of the flash-lag effect was reduced when a brief sound was presented synchronously with the flash or when the sound was presented before the flash, whereas the effect increased when the sound was presented after the flash. Both of the above-mentioned studies suggested that auditory temporal information can redefine the timing when a visual event (flash or bounce) occurs and can, consequently, modulate the spatial position of visual moving objects. Thus, it could be argued that auditory temporal information can also alter the perceived temporal offset position of a visual motion stimulus, resulting in the modulation of the magnitude of visual RM. Therefore, the present study focused on the offset timings of auditory information relative to visual motion.

It is important to note that in the two aforementioned studies (Heron et al., 2004; Vroomen \& de Gelder, 2004), audiovisual interaction occurred on the basis of the likely relation of a spatiotemporal characteristic between auditory and visual stimuli. Specifically, a brief burst of sounds appears to combine with a transient visual signal, visual bounce (Heron et al., 2004), and an abrupt flash (Vroomen \& de Gelder, 2004). Previous studies have shown that multiple sensory signals are likely to be bound into a single unified percept if they have some consistencies in one or more dimensions, such as space, time, and semantic content (Welch, 1999; Welch \& Warren, 1980; see Spence, 2007, for a review). The present study used visual smooth motion, which is often used in RM experiments. In the case of such visual motion, it can be assumed that the corresponding auditory stimulus would be a long-lasting sound, because the auditory stimulus has a consistency with the visual stimulus in the temporal dimension. Thus, a long-lasting sound was mainly used in this study (Experiments 1, 3, and 4), although a brief sound was tested as well (Experiment 2).

In Experiment 1, a visual stimulus moved horizontally for 1,200 msec and disappeared without warning, accompanied by a long-lasting sound. Various asynchronies in offset were introduced between the visual motion and the sound. Here, it was expected that the delayed and preceded offset of the sound, relative to the visual motion, would increase and decrease the visual RM, respectively, because such sounds would make the perceptual system misregister the temporal offset of visual motion. In Experiment 2, the visual stimulus moved horizontally for several milliseconds without any sound and disappeared at unpredictable positions. A brief sound, instead of a long-lasting one, was presented around the offset of the visual motion. The aim of this experiment was to investigate whether the transient signal generated by the offset of the long-lasting sound is essential for the effects found in Experiment 1. Experiments 3 and 4 investigated how the magnitude of visual RM is affected by the audiovisual interactions before the offsets of the long-lasting sound and visual motion were presented. For these purposes, various onset differences were introduced between the long-lasting sounds and the visual motion, together with some offset differences.

\section{EXPERIMENT 1}

The effect of long-lasting sounds on visual RM was investigated in this experiment. The onset of the sound was always synchronized with that of visual motion, but the offsets of auditory motion and of visual motion were not synchronized (i.e., auditory motion stopped before or extended beyond the end of visual motion). Specifically, 
the offset of the sounds was delayed (subsequent-offset block), relative to the offset of the visual motion stimulus, or preceded it (prior-offset block). It could be predicted that auditory temporal information can modulate the timing when a visual event occurs and, consequently, can modulate the spatial position of the moving visual object (Heron et al., 2004; Vroomen \& de Gelder, 2004). Therefore, it was expected that the sound would have an impact on the time at which the visual motion vanished in the visual RM situation, in which case, the delayed offsets of the sound should increase the visual RM and the preceded offset should reduce the visual RM.

\section{Method}

Participants. Five people between 18 and 32 years of age ( 1 female and 4 males) participated. All the participants had normal or corrected-to-normal vision and normal hearing. All the participants, except for the first author, were unaware of the purpose of the experiment. An informed consent was obtained from each participant before the experiment. All procedures were approved by the Ethics Committee for Faculty of Sciences, Letters, and Arts, Tohoku Uni- versity. These criteria apply for all the other experiments described below.

Apparatus and Materials. Visual stimuli were displayed on a 22-in. computer monitor (Mitsubishi, RHF223H, $75 \mathrm{~Hz}$ in refresh rate), controlled by an IBM-compatible personal computer (Dell XPS630) and MATLAB Psychophysics Toolbox extensions (Brainard, 1997; Pelli, 1997). A red circle $\left(14.2 \mathrm{~cd} / \mathrm{m}^{2}, 1.0^{\circ}\right.$ in diameter $)$ was displayed against a gray background $\left(13.6 \mathrm{~cd} / \mathrm{m}^{2}\right)$ as a fixation point. A white square (visual target, $62.8 \mathrm{~cd} / \mathrm{m}^{2}, 0.5^{\circ} \times 0.5^{\circ}$ ) appeared $11^{\circ}-12^{\circ}$ laterally, to either the left or the right of the display (around the boundary of the gray background) and $2.5^{\circ}$ below the horizontal median (Figure 1). Immediately after its onset, the visual target moved toward the center of the display at a speed of $7.5 \mathrm{deg} /$ $\mathrm{sec}$ for 1,200 msec. Five hundred milliseconds after the visual target vanished, a white probe $\left(62.8 \mathrm{~cd} / \mathrm{m}^{2}, 0.5^{\circ} \times 0.5^{\circ}\right)$ appeared $1.0^{\circ}$ above ${ }^{1}$ and $-0.9^{\circ}$ to $1.5^{\circ}$ (in steps of $0.15^{\circ}$ ) laterally, relative to the vanishing position of the visual target (negative values indicate that the probe was presented backward from the motion direction from the actual vanishing position of the visual target, whereas the positive values indicate that the probe was presented forward in the motion direction from the actual vanishing position of the visual target).

A long-lasting complex tone (triangle wave with a fundamental frequency of $400 \mathrm{~Hz}$; 20 -msec ramp for both its onset and off-

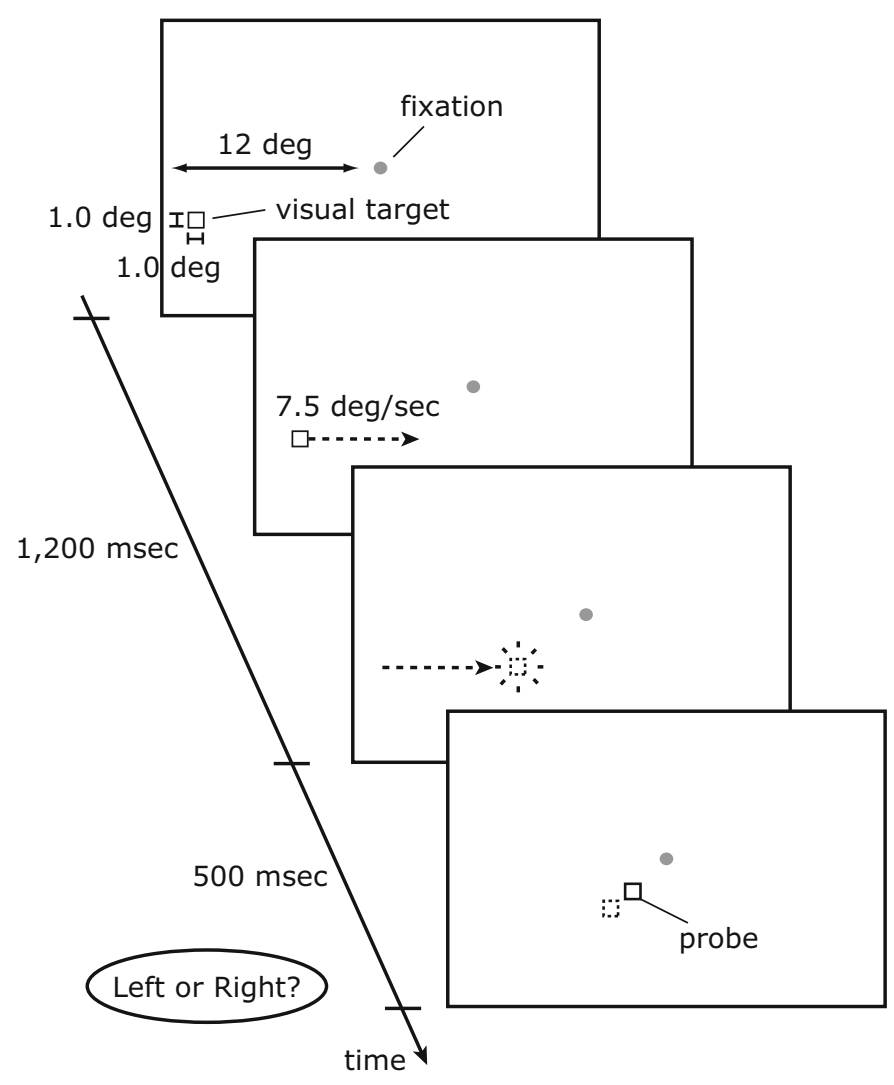

Figure 1. An example of the visual display and its sequence of presentation. While participants fixated at a fixation point, a white square (target) moved laterally from either side of the CRT display to its center at a speed of $7.5 \mathrm{deg} / \mathrm{sec}$ for $1,200 \mathrm{msec}$ and disappeared at an unpredictable position before the fixation. Five hundred milliseconds after the disappearance of the target, a probe was presented. The participants' task was to judge which side of the final position of the target the probe was presented on-left or right. On some trials, a long-lasting sound was additionally presented via headphones from the beginning of the target's motion, and the sound disappeared before, at, or after the disappearance of the visual target. 


\section{A}

Subsequent-offset block

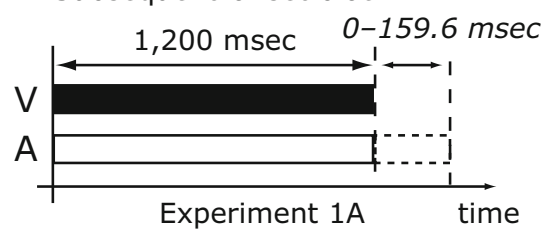

Prior-offset block

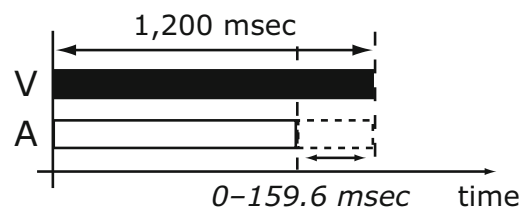

C

\section{Subsequent-offset block}

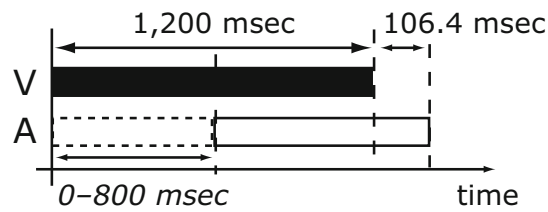

Prior-offset block

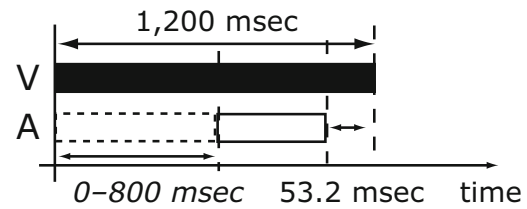

B

Subsequent-offset block

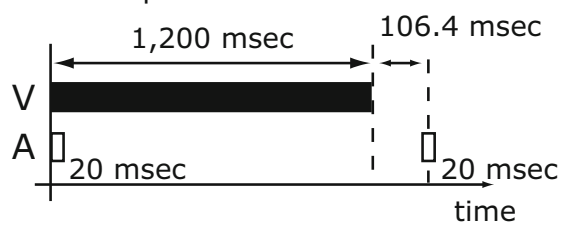

Prior-offset block

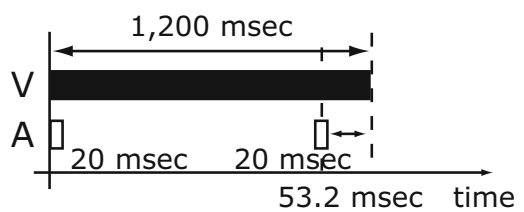

D

Subsequent-offset block

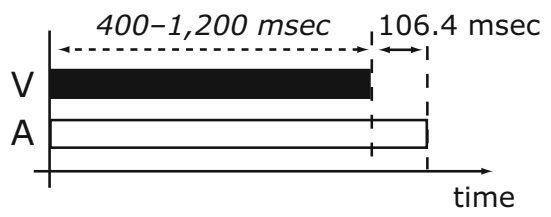

Prior-offset block

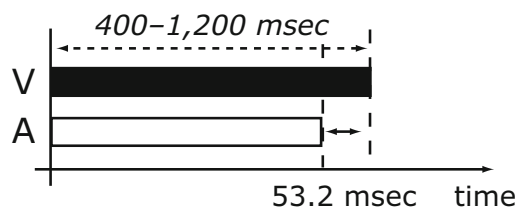

Figure 2. A schematic diagram of the experimental design for each experiment in the present study. (A) In Experiment 1, the relative offset timing of a long-lasting sound to visual motion was manipulated from 0 to $+159.6 \mathrm{msec}$ and from -159.6 to $0 \mathrm{msec}$, in the subsequent-offset and prioroffset blocks, respectively. (B) In Experiment 2, a short sound (20 msec) was presented at the onset of visual motion and $96.4 \mathrm{msec}$ after (the subsequent-offset block) or $43.2 \mathrm{msec}$ before (the prioroffset block) the offset of the visual motion. Experiment 2 also included long-lasting sound trials (see panel A) where a long-lasting sound was presented and withdrawn $106.4 \mathrm{msec}$ after (subsequentoffset block) or $53.2 \mathrm{msec}$ before (prior-offset block) the offset of visual motion. (C) In Experiment 3, onset asynchronies were introduced between the visual motion and the sound, in addition to the offset asynchronies $(+\mathbf{1 0 6 . 4} \mathbf{m s e c}$ in the subsequent-offset block and $\mathbf{- 5 3 . 2} \mathbf{m s e c}$ in the prior-offset block). (D) In Experiment 4, the exposure duration of the audiovisual stimuli was manipulated in steps of $400 \mathrm{msec}$ from 400 to $1,200 \mathrm{msec}$, whereas the offset asynchronies remained constant $(+106.4 \mathrm{msec}$ in the subsequent-offset block and $\mathbf{- 5 3 . 2} \mathrm{msec}$ in the prior-offset block).

set; sound pressure level of $83 \mathrm{~dB}$ ) was presented via headphones (Sennheiser, HDA200). The onset of the sound was always synchronized with that of the visual target. The amount of stimulus offset asynchrony (SOffA) between the sound and the visual target varied from 0 to $+159.6 \mathrm{msec}$ and from 0 to $-159.6 \mathrm{msec}$ in the subsequent-offset and prior-offset blocks, respectively, in steps of $53.2 \mathrm{msec}$ (negative SOffAs indicate that the sound lasted less long than the visual target, whereas positive SOffAs indicate that the sound lasted longer than the visual target). Thus, the duration of the sound was from 1,200 msec $\pm 0-159.6 \mathrm{msec}$ (Figure 2A).

Procedure. Three positive and negative SOffAs were tested in the subsequent-offset and prior-offset blocks, respectively. Both blocks included trials with zero SOffA and no sound (silent condition). Thus, five sound conditions were compared in each block (i.e., $0,+53.2$,
+106.4 , and $+159.6 \mathrm{msec}$ of SOffA and no-sound conditions for the subsequent-offset block, and $0,-53.2,-106.4$, and $-159.6 \mathrm{msec}$ of SOffA and no-sound conditions for the prior-offset block).

The participants viewed the display binocularly from a distance of $57 \mathrm{~cm}$. They were asked to maintain fixation during the presentation of the visual target and to report whether the position of the probe was to the left or the right of the vanishing position of the visual target by pressing the appropriate button on the computer keyboard (two-alternative forced choice). The amount of lateral displacement of the probe from the actual vanishing position of the visual target varied from trial to trial according to a staircase method (Cornsweet, 1962). ${ }^{2}$ There were 4 staircases for each sound condition: 2 (motion direction of the visual target: rightward or leftward motion) $\times$ 2 (order of staircase: ascending or descending series). Each staircase 


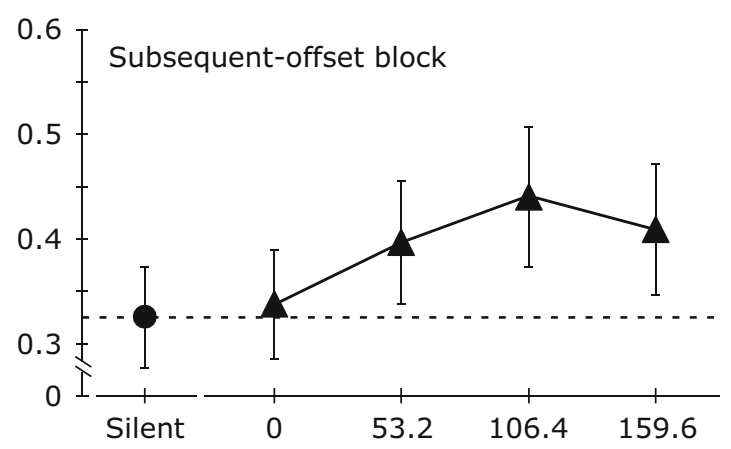

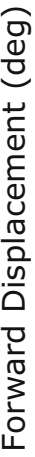

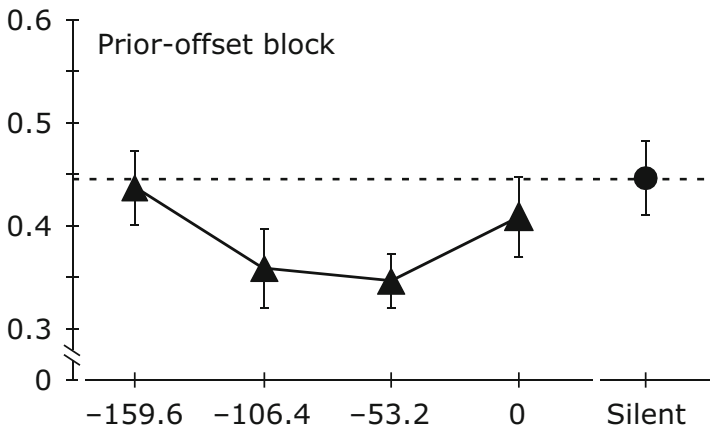

Offset Difference Between Sound and Visual Motion (msec)

Figure 3. Results of Experiment 1. The horizontal axis indicates temporal offset differences between the long-lasting sound and visual motion (positive and negative values indicate that the sound disappeared after and before the disappearance of the visual motion, respectively). The vertical axis indicates the magnitude of forward displacement of the final position of the moving visual target in the direction of motion. The horizontal dashed line shows the magnitude of representational momentum for the no-sound condition (i.e., baseline). Standard errors are shown as error bars.

was terminated after 5 reversals of the response sequence. Thus, 20 reversals were obtained from 4 staircases in each condition. A subjective vanishing position of the visual target for each condition was obtained, averaging across 16 reversal points after the first reversal for each staircase was discarded. The order of the 20 staircases (4 staircases for each of five sound conditions) randomly varied for each participant. The order of the blocks was counterbalanced among the participants.

\section{Results and Discussion}

Differences between the actual and subjective final positions of the visual target (the magnitude of RM) were averaged across participants for each condition, as is shown in Figure 3. A positive value indicates the displacement of the visual target in the direction of visual motion, and a negative value indicates the displacement in the direction opposite from visual motion. Zero reflects no displacement. The one-tailed $t$ tests revealed that the averages of displacements for all the conditions were significantly higher than zero $[t \mathrm{~s}(4)>7.20, p \mathrm{~s}<.002$, effect size $r \mathrm{~s}>$ .964 , for the subsequent-offset block, and ts $(4)>9.50$, $p \mathrm{~s}<.001, r \mathrm{~s}>.979$, for the prior-offset block]. Thus, it was confirmed that RM (forward displacements) occurred in all the conditions. The magnitudes of RM were analyzed in a one-way repeated measure ANOVA for each block. The main effect in the sound condition was observed for each block $\left[F(4,16)=6.63, p=.002, \eta^{2}=\right.$ .184 , for the subsequent-offset block, and $F(4,16)=4.02$, $p=.019, \eta^{2}=.293$, for the prior-offset block]. A multiple comparison (Tukey's HSD method, $\alpha<.05$ ) revealed that the magnitude of RM was significantly larger for $+106.4 \mathrm{msec}\left(0.48^{\circ}\right)$ and $+159.6 \mathrm{msec}\left(0.46^{\circ}\right)$ of SOffA for the subsequent-offset block $\left(M S_{\mathrm{e}}=0.008\right)$ and significantly smaller for $-53.2 \mathrm{msec}\left(0.36^{\circ}\right)$ of SOffA for the prior-offset block $\left(M S_{\mathrm{e}}=0.012\right)$, as compared with that for the silent condition. Thus, when the sound lasted longer than the visual motion (subsequent-offset block), the magnitude of RM increased with the increasing SOffA. When the sound lasted less long than the visual motion (prior-offset block), on the other hand, the magnitude of RM decreased at -53.2 msec of SOffA and gradually returned to the magnitude of RM without sound.

One would conjecture that some decisional biases might contribute to the effects. For example, because auditory offsets on most trials in the prior-offset block were prior to visual offsets and auditory offsets on most trials in the subsequent-offset block were subsequent to visual offset, it might be possible that this manipulation might introduce biases (anticipation, habituation, and so on). ${ }^{3}$ However, the results showed that the magnitude of the visual RM changed as a function of the auditory offset (a convex upward function with a maximum at $+106.4 \mathrm{msec}$ for the subsequent-offset block and a convex downward function with a minimum at $-53.2 \mathrm{msec}$ for the prior-offset block). These biases cannot account for the functions. In the case in which the blocked design would bias the uniform judgments or responses on the basis of the existence of the auditory offset itself, the magnitude of the visual RM should have been consistent across the auditory offset conditions. Otherwise, if the offset timing of the auditory stimuli induced decisional biases for the large offset, triggering a larger offset of the visual motion stimulus, the maximum effect of the auditory offset should have been observed at the maximum auditory offset for each block.

Although evidence was given for the effect of auditory information on the visual RM in this experiment, it is not clear how auditory information could modulate visual RM. As was mentioned in the introduction, long-lasting sounds were arbitrarily used as an auditory counterpart to smooth visual motion in order for the participants to easily assume that the auditory and visual signals originated from a common external event. However, it is not clear whether such long-lasting sounds were necessary. Alternatively, one could argue that only a transient signal generated by the offset of the sound might have been crucially responsible for the phenomenon, similar to the manner in which transient auditory signals capture the temporal aspects of visual stimuli in temporal ventriloquism phenomena (Morein-Zamir et al., 2003; Vroomen \& de Gelder, 2004). In this case, the results of Experiment 1 should be replicated with a brief transient sound presented around the offset of visual motion. This possibility was tested in Experiment 2. 


\section{EXPERIMENT 2}

The possibility was tested that a transient signal generated by the offset of the long-lasting sound might affect the judgments of the vanishing position of the visual target in Experiment 1. For this purpose, a brief sound was presented either before (prior-offset block) or after (subsequent-offset block) the offset of the visual target. If the transient signal accompanied by the offset of the long-lasting sound is a crucial factor for the auditory effect on the visual RM observed in Experiment 1, the same results as those in Experiment 1 should be obtained with the brief sound as well. In this experiment, another brief sound was presented, synchronized with the onset of the visual motion in order to ensure the association between the auditory and visual stimuli, as in Experiment 1.

\section{Method}

Participants. The 5 people who had participated in Experiment 1 also participated in this experiment (i.e., 4 volunteers and 1 of the authors).

Apparatus and Materials. The apparatus and visual stimuli were the same as those used in Experiment 1. A brief complex tone was prepared (20-msec triangle wave with a fundamental frequency of $400 \mathrm{~Hz}$; $10-\mathrm{msec}$ ramp for both its onset and offset; sound pressure level of $83 \mathrm{~dB}$ ), in addition to the long-lasting sound used in Experiment 1.

Procedure. Three conditions - brief sound, long-lasting sound, and silent conditions - were compared. In the brief sound condition, the brief complex tone was presented twice in a trial. The first sound was presented at the beginning of the visual target, and the second sound was presented either $96.4 \mathrm{msec}$ after or $43.2 \mathrm{msec}$ before the offset of the visual target, such that the peak amplitude of the second sound came $106.4 \mathrm{msec}$ after or $53.2 \mathrm{msec}$ before the offset of the visual target (Figure 2B). In the long-lasting sound condition, the longlasting sound was presented with either $+106.4 \mathrm{msec}$ or $-53.2 \mathrm{msec}$ of SOffA. In order to reduce the number of trials, these two offset timings were used because +106.4 and $-53.2 \mathrm{msec}$ of SOffA had exhibited the largest effects in the subsequent-offset and prior-offset blocks, respectively, in Experiment 1. These two timings were tested in two separate sets of blocks, as in Experiment 1. The trials on which the second brief sound appeared after the visual target vanished and those on which the long-lasting sound was presented with the SOffA of $+106.4 \mathrm{msec}$ were conducted in the subsequent-offset block. The trials in which the second brief sound appeared before the visual target vanished and those on which the long-lasting sound was presented with the SOffA of $-53.2 \mathrm{msec}$ were conducted in the prioroffset block. The staircase method was used, as in Experiment 1. The order of the 12 staircases (4 staircases for each of the three sound conditions) randomly varied for each participant. The order of the blocks was counterbalanced among the participants.

\section{Results and Discussion}

The magnitude of RM was averaged across participants for each condition, as is shown in Figure 4. The $t$ tests revealed that the averages of displacements for all the conditions were significantly higher than zero $[t \mathrm{~s}(4)>7.54, p \mathrm{~s}<$ $.002, r \mathrm{~s}>.967$, for the subsequent-offset block, and $t \mathrm{~s}(4)>$ $8.82, p \mathrm{~s}<.001, r \mathrm{~s}>.975$, for the prior-offset block]. Thus, it was confirmed that RM occurred in all the conditions. A one-way repeated measures ANOVA for each block revealed the main effect of sound condition $[F(2,8)=8.27, p=.011$, $\eta^{2}=.209$, for the prior-offset block, and $F(2,8)=6.38, p=$ $.022, \eta^{2}=.101$, for the subsequent-offset block]. A multiple comparison (Tukey's HSD method, $\alpha<.05, M S_{\mathrm{e}}=0.013$ for
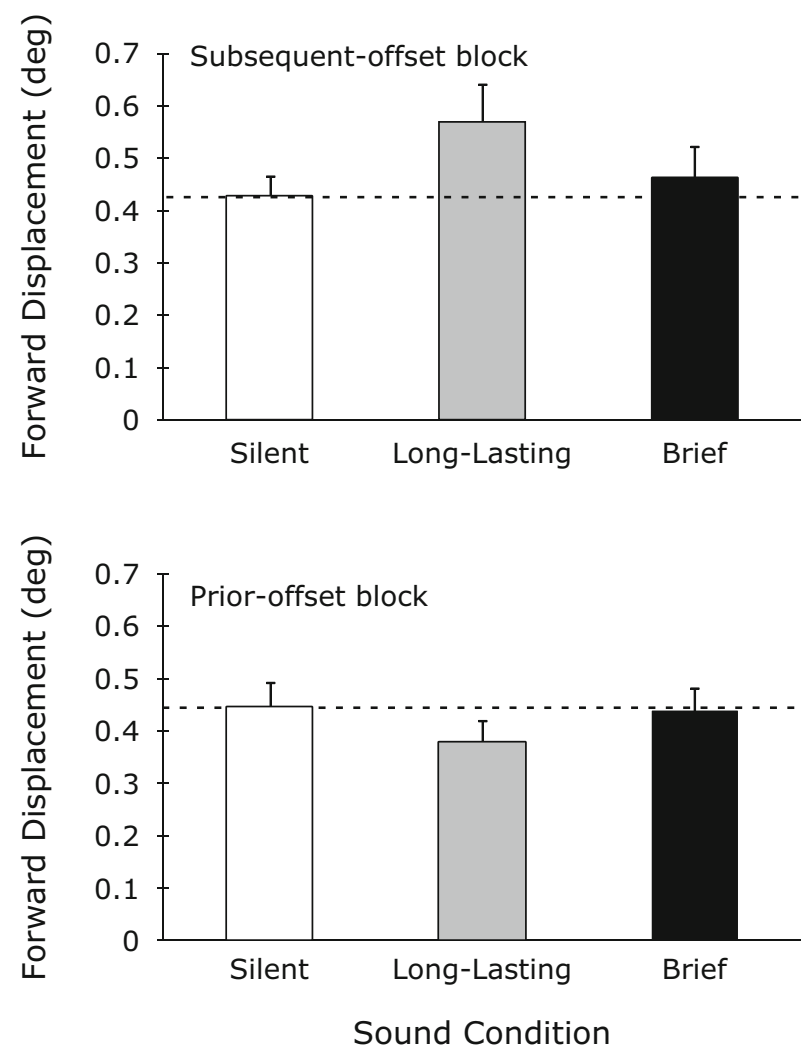

Figure 4. Results of Experiment 2. For short-sound trials, a short sound $(20 \mathrm{msec})$ was presented at the onset of visual motion and $96.4 \mathrm{msec}$ after (subsequent-offset block) or $43.2 \mathrm{msec}$ before (prior-offset block) the offset of the visual motion. For long-lasting sound trials, a long-lasting sound was presented and withdrawn 106.4 msec after (subsequent-offset block) or $53.2 \mathrm{msec}$ before (prior-offset block) the offset of visual motion. The horizontal dashed line shows the magnitude of representational momentum for the no-sound condition (i.e., baseline). Standard errors are shown as error bars.

the prior-offset block, and $M S_{\mathrm{e}}=0.004$ for the subsequentoffset block) revealed that the long-lasting sound led to significantly larger and smaller visual RM for the prior-offset and subsequent-offset blocks, respectively, replicating the results in Experiment 1. In contrast, the alteration of the visual RM was no longer observable with the presentation of the brief sound alone. These results indicate that the effect of sounds on the visual RM observed in Experiment 1 was not likely to be attributable only to transient signals generated by sounds. This, in turn, indicates that more crucial audiovisual interactions occur before the offset arrives.

\section{EXPERIMENT 3}

On the basis of the results of Experiment 2, it was hypothesized that a close association between auditory and visual information, based on the onset synchronization, might be essential for the present audiovisual interaction. Previous studies have shown that spatial coincidence and temporal synchrony are two key factors for information from several modalities to be integrated or interactive (Spence, 2007; Welch, 1999; Welch \& Warren, 1980). For 
example, in the spatial ventriloquism literature, the magnitude of visual capture of a sound decreased as the spatial and/or temporal distance between the visual and auditory stimuli increased or even disappeared, due to the introduction of some temporal asynchronies in audiovisual signals (Jack \& Thurlow, 1973; Radeau \& Bertelson, 1987; Slutsky \& Recanzone, 2001). In particular, with regard to motion perception, any temporal correspondence in the onset between auditory and visual signals facilitates audiovisual interactions. Soto-Faraco, Lyons, Gazzaniga, Spence, and Kingstone (2002) reported that visual motion captured the direction of auditory motion when their onsets were synchronized, but not when visual motion was presented as lagging $500 \mathrm{msec}$ behind the auditory motion. Sanabria, Soto-Faraco, Chan, and Spence (2004) extended the previous findings. They demonstrated that when the onsets of visual and auditory motion were consistent, visual motion could capture the direction of auditory motion, irrespective of the offset asynchronies. In contrast, when the onsets of visual and auditory motion were inconsistent, visual motion could not capture the direction of auditory motion even if the offsets were synchronized. These results suggest that a close association between auditory and visual signals, triggered by their onset synchrony, might play an essential role in the present audiovisual interaction. Hence, the onset asynchrony was introduced between the sound and visual target in Experiment 3 in order to eliminate the association between the auditory and visual stimuli (Figure 2C). If the onset synchrony contributed to the association between auditory and visual signals, no effect of the sound on visual RM should be observed when the onsets of these stimuli were asynchronously presented.

The temporal range within which audiovisual interactions can be observed varied with the type of stimuli used. In order not to associate between audiovisual stimuli, the onset asynchronies of audiovisual stimuli introduced in Experiment 3 should be long enough to be beyond this range. For example, for a double-flash illusion, where multiple auditory stimuli could change the perception of a static visual flash as multiple ones, the auditory effect was reduced to less than half to around $\pm 150 \mathrm{msec}$ (Shams, Kamitani, \& Shimojo, 2000). In a stream-bounce display (Sekuler, Sekuler, \& Lau, 1997), in which two visual stimuli moved toward and then across one another, the effect of a transient auditory stimulus inducing dominant bouncing perception was weak or eliminated when the auditory stimulus was presented $300 \mathrm{msec}$ before or after the visual stimuli overlapped (Watanabe \& Shimojo, 2001). Considering these studies, the present experiment adopted 400 and $800 \mathrm{msec}$ of onset asynchrony, where the association between auditory and visual stimuli would not be expected to be constructed.

\section{Method}

Participants. The 5 people who had participated in Experiment 1 also participated in this experiment (i.e., 4 volunteers and 1 of the authors).

Apparatus and Materials. The apparatus and visual stimuli were the same as those in Experiment 1. Four sound conditions were compared: onset synchrony, two onset asynchronies, and silent conditions. For the onset synchrony condition, the onset of the long- lasting sound and the visual target were synchronized with each other, as in Experiments 1 and 2. For the onset asynchrony conditions, two stimulus onset asynchronies (SOAs) between the sound and the visual target were tested: 400 and $800 \mathrm{msec}$ (Figure 2C). For the onset synchrony and onset asynchrony conditions, the SOffA was either +106.4 or $-53.2 \mathrm{msec}$. These SOffAs were the same as those in Experiment 2. Thus, the exposure duration of the sound was $1,200 \mathrm{msec}+$ SOffA for the onset synchrony conditions, and $1,200 \mathrm{msec}-\mathrm{SOA}+$ SOffA for the onset asynchrony conditions. Except for these parameters mentioned above, the other physical characteristics of the sound were the same as those in Experiment 1. It should be noted that the exposure duration of the visual target was always 1,200 msec.

Procedure. The positive SOffA was tested in the subsequentoffset block, and the negative SOffA was tested in the prior-offset block. Both blocks included trials with no sound (silent condition). The order of the blocks was counterbalanced among the participants. The staircase method was used as in Experiments 1 and 2. The order of the 16 staircases (4 staircases for each of four sound conditions) randomly varied for each participant. The order of the blocks was counterbalanced among the participants.

\section{Results and Discussion}

The magnitude of RM was averaged across participants for each condition, as shown in Figure 5. The $t$ tests revealed that the averages of displacements for all the conditions were significantly higher than zero $[t \mathrm{~s}(4)>7.05$,
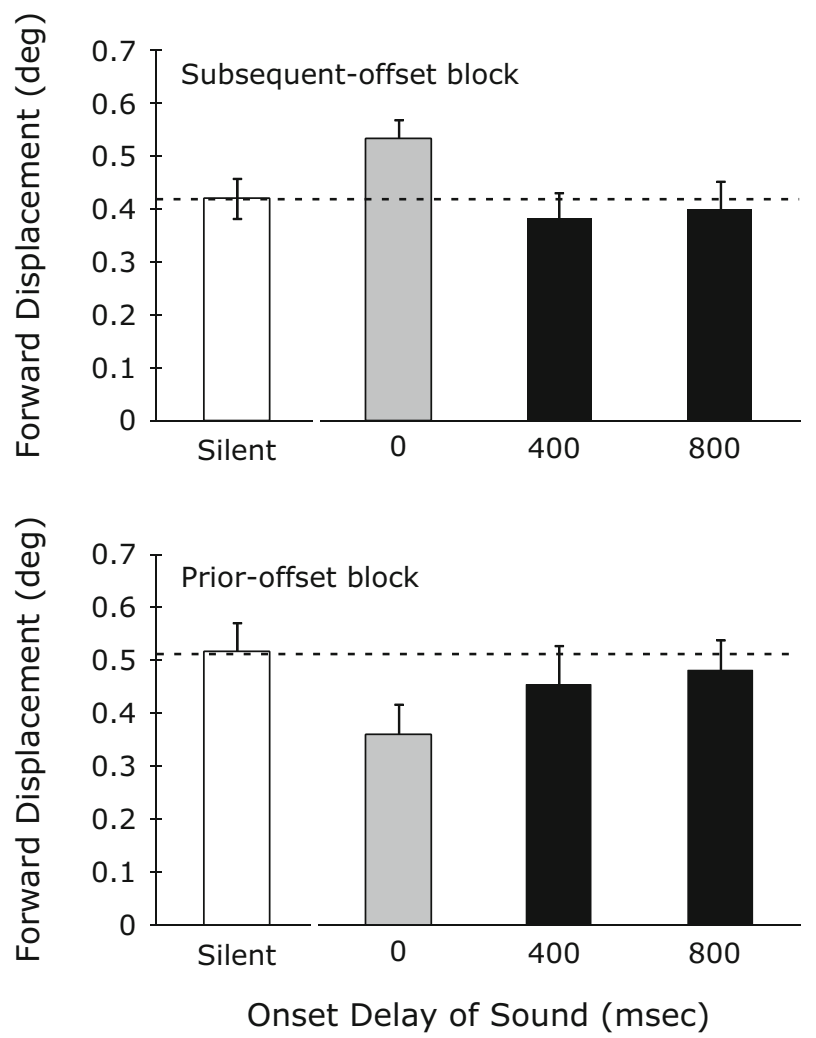

Figure 5. Results of Experiment 3. Onset asynchronies were introduced between the visual motion and the sound, in addition to the offset asynchronies $(+106.4 \mathrm{msec}$ in the subsequent-offset block and $\mathbf{- 5 3 . 2} \mathbf{m s e c}$ in the prior-offset block). The horizontal dashed line shows the magnitude of representational momentum for the no-sound condition (i.e., baseline). Standard errors are shown as error bars. 
$p \mathrm{~s}<.003, r \mathrm{~s}>.962$, for the subsequent-offset block, and $t \mathrm{~s}(4)>6.02, p \mathrm{~s}<.004, r \mathrm{~s}>.949$, for the prior-offset block]. Thus, it was confirmed that RM occurred in all the conditions. The subsequent-offset and prior-offset blocks were separately analyzed. A one-way repeated measures ANOVA for each block revealed a main effect of the sound condition $\left[F(3,12)=9.74, p=.002, \eta^{2}=\right.$ .295 for the subsequent-offset block, and $F(3,12)=6.66$, $p=.007, \eta^{2}=.178$ for the prior-offset block]. A multiple comparison (Tukey's HSD method, $\alpha<.05$ ) revealed that only the sound in the onset synchrony condition resulted in a significantly larger and smaller visual RM for the subsequent-offset and prior-offset blocks, respectively, than did that in the silent conditions, replicating the results in Experiment $1\left(M S_{\mathrm{e}}=0.010\right.$ for the subsequent-offset block, and $M S_{\mathrm{e}}=0.014$ for the prior-offset block). However, at 400 or $800 \mathrm{msec}$ of SOA, the sounds did not alter the visual RM anymore, although the sounds had the most effective SOffAs, as was shown in Experiment 1. These results suggest that the introduction of SOffAs between the sounds and visual motion was not sufficient to evoke the audiovisual interaction on a visual RM. Instead, the onset synchrony of audiovisual signals played an essential role in triggering the interaction that occurred at their offset. However, one matter of concern was that the introduction of the onset asynchrony of audiovisual signals simultaneously decreased the exposure duration of the sounds. Thus, this decrease in exposure duration could be considered to reduce the effect of the sound on the visual RM. This possibility was tested in the next experiment.

\section{EXPERIMENT 4}

This experiment investigated whether the main factor that caused a reduction in the effect of sounds on visual RM in Experiment 3 was the onset synchrony of audiovisual signals or the exposure duration of the sound. For this purpose, the exposure duration of sounds was manipulated, whereas the SOA between audiovisual stimuli was always zero and the SOffA was either +106.4 or $-53.2 \mathrm{msec}$ (see Figure 2D). If the exposure duration of the sound played a crucial role in Experiment 3, the magnitude of the visual RM should change, depending on the duration of the sound. In contrast, if the onset synchrony of audiovisual signals was a more important factor, the magnitude of the visual RM should be constant across the duration of the sound.

\section{Method}

Participants. The 5 people who had participated in Experiment 1 also participated in this experiment (i.e., 4 volunteers and 1 of the authors).

Apparatus and Materials. The apparatus was the same as that in Experiment 1 . Three exposure durations of the visual stimulus were tested: 400,800 , and 1,200 msec (Figure 2D). For each of the exposure duration conditions, the SOA between audiovisual stimuli was always zero, and the SOffA was either +106.4 or $-53.2 \mathrm{msec}$. These SOffAs were the same as those in Experiment 2. Thus, the exposure durations of the sound corresponded with those in Experiment 3.

The offset position of the visual target for each condition was the same as that in Experiments 1, 2, and 3-that is, in the range between $2.0^{\circ}$ and $3.0^{\circ}$ from the fixation - and its onset was changed depending on the exposure time: $11^{\circ}-12^{\circ}$ from the fixation for $1,200 \mathrm{msec}$ of the exposure duration, $8^{\circ}-9^{\circ}$ for $800 \mathrm{msec}$, and $5^{\circ}-6^{\circ}$ for $400 \mathrm{msec}$.

Procedure. The positive SOffA was tested in the subsequentoffset block, and the negative SOffA was tested in the prior-offset block. Both blocks included trials with no sound (silent condition). The exposure time for the silent condition was kept constant, consistent with Experiment 3. The staircase method was used as in the former three experiments. The order of the 16 staircases (4 staircases for each of four sound conditions) randomly varied for each participant. The order of the blocks was counterbalanced among the participants.

\section{Results and Discussion}

The magnitude of RM was averaged across the participants for each condition, as shown in Figure 6. The $t$ tests revealed that the averages of displacements for all the conditions were significantly higher than zero $[\operatorname{ts}(4)>7.47$, $p \mathrm{~s}<.002, r \mathrm{~s}>.967$, for the subsequent-offset block, and $t \mathrm{~s}(4)>5.17, p \mathrm{~s}<.007, r \mathrm{~s}>.933$, for the prior-offset block]. Thus, it was confirmed that RM occurred in all the conditions. A one-way repeated measure ANOVA for each block revealed a main effect of sound condition $\left[F(3,12)=15.68, p<.001, \eta^{2}=.304\right.$, for the subsequent-
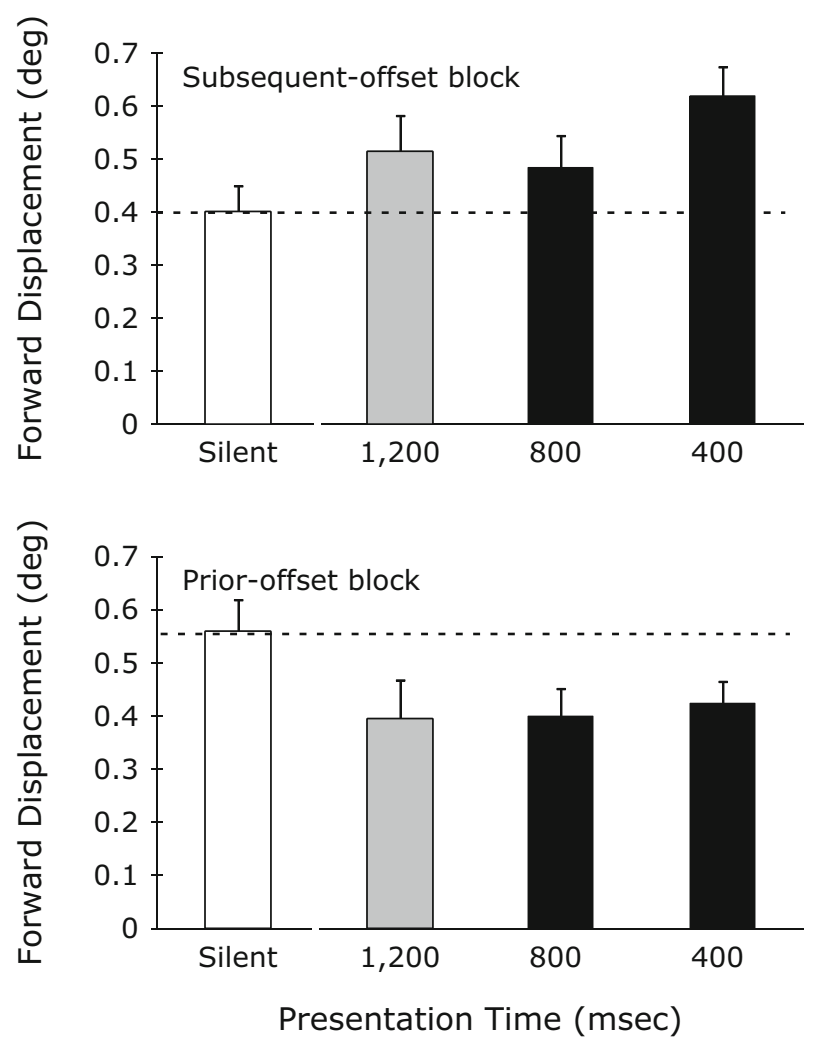

Figure 6. Results of Experiment 4. The exposure duration of audiovisual stimuli was manipulated in steps of $400 \mathrm{msec}$ from 400 to 1,200 msec (horizontal axis), whereas the offset asynchronies remained constant $(+106.4$ msec in the subsequent-offset block and $\mathbf{- 5 3 . 2} \mathbf{m s e c}$ in the prior-offset block). The horizontal dashed line shows the magnitude of representational momentum for the no-sound condition (i.e., baseline). Standard errors are shown as error bars. 
offset block, and $F(3,12)=5.90, p=.010, \eta^{2}=.262$, for the prior-offset block]. A multiple comparison (Tukey's HSD method, $\alpha<.05$ ) revealed that the presentation of the sound with its onset synchronized with that of the visual motion enhanced the magnitude of the visual RM in the subsequent-offset block and reduced the magnitude of the visual RM in the prior-offset block, irrespective of the exposure duration of the audiovisual stimuli $\left(M S_{\mathrm{e}}=\right.$ 0.010 for the subsequent-offset block and $M S_{\mathrm{e}}=0.021$ for the prior-offset block). These results indicate that a more crucial factor in the reduction of the effect of sound on the visual RM in Experiment 3 was the asynchrony in the onset of the audiovisual stimuli, and not their exposure duration. The synchrony of signals from various sensory modalities is one of the key factors for associating the information from one modality with the other, as indicated by previous studies (Jack \& Thurlow, 1973; Radeau \& Bertelson, 1987; Slutsky \& Recanzone, 2001). This is the case for the audiovisual interaction on the visual RM. The data show that the close association between the auditory and visual signals is necessary in order for the auditory temporal offset information to affect the visual RM.

\section{GENERAL DISCUSSION}

In the present study, four experiments were conducted to address the question of whether inputs from the auditory modality can influence the localization of the final position of moving visual objects. The basic findings of our study are as follows. The visual RM was enhanced when the sound was continuously presented from the beginning of the visual motion and lasted longer than the visual motion. On the other hand, the visual RM was reduced when the sound was terminated shortly before the visual motion. The amount of enhancement and reduction changed depending on the difference in the offset timing between the sound and visual motion, indicating the temporal window of the audiovisual interaction for this phenomenon (ca. 50-100 msec), as shown in Experiment 1 . The presentation of a brief transient sound alone before or after the offset of a visual motion did not lead to the alteration of the visual RM. This result indicates that the transient signal contained in the offset of the longlasting sound in Experiment 1 was not the main factor for this phenomenon (Experiment 2). The introduction of asynchronies in the onset between the auditory and visual stimuli eliminated the effect of the sound on the visual RM (Experiment 3). In contrast, the modulation of the auditory stimuli on the visual RM was observed again when the onset was synchronized between the auditory and visual stimuli, regardless of the difference in the stimulus duration $(400,800$, and 1,200 msec), as shown in Experiment 4 . These results suggest that the close association between the auditory and visual signals accomplished by their onset synchrony is a necessary condition for the effect of the sounds on the visual RM.

\section{Implications for RM Research}

Previous studies have shown that the final position of a moving stimulus is mislocalized in the direction of motion in each of the auditory and visual modalities. However, the effect of auditory information on visual RM has not been reported, although there is a study showing the effect of linear frequency changes of sounds on visual representational gravity (Hubbard \& Courtney, 2010). In the present experiments, the effects of auditory information on the visual RM are clearly revealed. These results suggest that the underlying mechanisms for the visual RM at least utilize the information from the auditory modality. It should be noted that the auditory stimulus used in the present study did not include any motion cue. All that was manipulated was the difference in the offset (and onset) timing between the visual and auditory stimuli, and it was definitely effective. Nagai and Saiki (2005) suggested that there was a possibility that a coding error of the offset timing of a visual motion stimulus - specifically, later coding of the offset timing than for the physical one-was one of the causes for the visual RM. Moreover, it was reported that, when participants could control the time at which a target vanished by pressing a button, the amount of visual RM decreased under a condition in which the offset of the moving visual stimulus lagged behind participants' intended vanishing timings (Jordan, Stork, Knuf, Kerzel, \& Müsseler, 2002). This finding indicates that the perceived offset position of the visual stimulus can be attracted to the intended moment of offset. Considering these notions and findings, it is likely that auditory information would modulate the coding of the offset timing of the visual motion, resulting in the increase or decrease in visual RM. However, it should also be noted that the offset timing of the visual motion stimulus was not completely replaced with that of auditory stimuli in the present study. For example, in the prior-offset blocks, where the sound disappeared earlier than the visual motion stimulus, the visual displacement always occurred in the forward direction of visual motion, but not in the opposite direction. Moreover, the sounds increased or reduced the visual RM only by $20 \%-30 \%$, as compared with the no-sound condition. These results suggest that the auditory temporal information has a modulatory effect on the visual RM.

Apart from the effect of audiovisual interaction on visual RM, it should be noted that reliable visual RMs were obtained in our study in spite of the presentation of smooth motion and a fixation point. The fixation point was presented to avoid the confounding effects of eye movements on the visual RM in the present experiments. It was reported that the maintenance of the fixation could reduce or eliminate the visual RM with smooth motion (Kerzel, 2000). Thus, our results contradict those of previous studies. Kerzel (2006) suggested that the goodness or quality of motion perception defined by various properties, such as the object's shape, the ISI, and the trajectory, contributes to the internal extrapolation process and the resulting visual RM. The best parameters for inducing a reliable amount of visual RM had been obtained in our pilot experiments. The spatiotemporal properties of visual stimuli (luminance, traveling distance, duration, size, and so on) used in the present study might produce an appropriate motion perception sufficient to trigger the internal extrapolation process and induce visual RM. Explicating 
this line of discussion, however, is beyond the scope of the present study.

\section{Implications for Audiovisual Interactions in the Temporal Domain}

As suggested by the modality appropriateness hypothesis (Welch \& Warren, 1980), in general, audition dominates vision in the temporal domain, whereas vision dominates audition in the spatial domain. With regard to the auditory dominance, most of the previous studies have focused on the audiovisual interaction of instantaneous or transient events such as abrupt onsets of flash and burst (auditory driving, Gebhard \& Mowbray, 1959; illusory flash, Shams, Kamitani, \& Shimojo, 2000, 2002; flashlag effect, Alais \& Burr, 2003, and Vroomen \& de Gelder, 2004; temporal order judgments, Morein-Zamir et al., 2003; apparent motion, Getzmann, 2007) and collisions (Heron et al., 2004; Sekuler et al., 1997). However, few studies have shown the audiovisual interactions on relatively long-lasting events, except for audiovisual speech studies (e.g., Dixon \& Spitz, 1980). The new findings on visual RM in this study suggest that sounds might modulate the duration or the persistence of the visual event or representation. This aspect of auditory temporal information has not been investigated intensively, although it is also essential, especially in cases in which visual signals are ambiguous or unavailable. For example, a visual object is often occluded by other visual objects, because of the 3-D nature of the visual world, or by our own body parts (i.e., rear space). In such cases, only a sound accompanied by the visual event can help us determine the persistence of the event. Thus, our findings are ecologically reasonable and could provide a new standpoint for audiovisual interactions.

On the other hand, it should be noted that a long-lasting sound will not influence visual motion processing unless the sensory signals are assumed to be sufficiently associated, as is shown in all the experiments in this study. These results can be interpreted on the basis of the wellknown unity assumption (Welch, 1999; Welch \& Warren, 1980). This assumption is made by participants (or the brain) when determining whether inputs from several modalities are attributable to a single external event. Spatial consistency and temporal synchrony of the inputs from various modalities are reported to be the most powerful variables for this assumption (Welch, 1999). For example, in the spatial ventriloquism literature, the magnitude of the visual capture of a sound decreased as the spatial distance between the visual and the auditory stimuli increased (Jack \& Thurlow, 1973) or even disappeared due to the introduction of some temporal asynchronies in the audiovisual signals (Jack \& Thurlow, 1973; Radeau \& Bertelson, 1987; Slutsky \& Recanzone, 2001). As was shown in Experiments 3 and 4, the onset synchrony of the audiovisual signals was found to be a powerful source for associating auditory and visual information in the present study as well.

Regarding the spatial domain, however, there was no consistency between the auditory and visual stimuli, because the sound used in the present study did not have any motion cues. The reason why the audiovisual interaction was not inhibited by this spatial inconsistency may be related to the fact that in the spatial domain, audition is often less reliable and less salient than vision. The auditory system is relatively insensitive to target motion. For example, the minimum movement angle for detecting motion in the auditory modality is $2^{\circ}-4^{\circ}$ (Harris \& Sergeant, 1971), $5^{\circ}$ (Grantham, 1986), or even more (Perrott \& Musicant, 1977), although the value depends on measurement methods and the stimulus parameters, such as angle in azimuth, duration, and so on. These threshold angles are much higher than those observed in the visual modality (at most 30 min; McKee \& Watamaniuk, 1994). Moreover, it was also reported that auditory motion information did not affect visual motion direction perception when visual motion information was obviously presented (Soto-Faraco, Spence, \& Kingstone, 2004). Accordingly, even if the sounds have motion information, the perceptual system would rarely utilize such relatively unreliable spatial information to code the position of visual stimuli in the present research. Thus, it can be speculated that audiovisual interactions might require three steps for the present effect of auditory temporal information on visual RM: (1) The onset synchrony of audiovisual signals helps to associate them, (2) the audiovisual association is maintained during visual motion, and finally, (3) the offset asynchronies of audiovisual signals alter the visual temporal offset and, consequently, modulate the spatial position of visual moving objects.

In conclusion, this study demonstrated a clear audiovisual cross-modal interaction on visual RM. Specifically, when a long-lasting sound was presented with its onset synchronized with that of the visual motion, longer sounds led to a larger visual RM, and shorter sounds led to a shorter RM. In contrast, there was no effect of sound on the visual RM when a brief sound was presented around the offsets of the visual motion or when asynchronous onsets (400 and $800 \mathrm{msec}$ ) were introduced. The results suggest that visual motion representation can be modulated by a sound if the close association of audiovisual information occurs.

\section{AUTHOR NOTE}

S.H. is now at the Department of Psychology, Rikkyo University. This research was supported by a Grant-in-Aid for Scientific Research to W.T., J.G., and Y.S. (No. 19001004) and by a Research Fellowship of the Japan Society for the Promotion of Science for Young Scientists to S.H. (No.19004400). Correspondence concerning this article should be addressed to W. Teramoto, Research Institute of Electrical Communication, Tohoku University, Katahira 2-1-1 Aoba-ku Sendai 980-8577, Japan (e-mail: teraw@ais.riec.tohoku.ac.jp).

\section{REFERENCES}

Alais, D., \& BURr, D. (2003). The "flash-lag" effect occurs in audition and cross-modally. Current Biology, 13, 59-63.

Bertelson, P., \& Aschersleben, G. (1998). Automatic visual bias of perceived auditory location. Psychonomic Bulletin \& Review, 5, 482489.

Brainard, D. H. (1997). The Psychophysics Toolbox. Spatial Vision, 10, 433-436.

Cornsweet, T. N. (1962). The staircase method in psychophysics. American Journal of Psychology, 75, 485-491. 
Dixon, N. F., \& SpITZ, L. (1980). The detection of auditory visual desynchrony. Perception, 9, 719-721.

Finke, R. A., Freyd, J. J., \& Shyi, G. C. (1986). Implied velocity and acceleration induce transformations of visual memory. Journal of Experimental Psychology: General, 115, 175-188.

FREYD, J. J., \& FinKE, R. A. (1984). Representational momentum. Journal of Experimental Psychology: Learning, Memory, \& Cognition, 10, $126-132$.

Freyd, J. J., \& FinKe, R. A. (1985). A velocity effect for representational momentum. Bulletin of the Psychonomic Society, 23, 443-446.

Freyd, J. J., Kelly, M. H., \& DeKay, M. L. (1990). Representational momentum in memory for pitch. Journal of Experimental Psychology: Learning, Memory, \& Cognition, 16, 1107-1117.

FröHLICH, F. W. (1923). Über die Messung der Empfindungszeit [On the measurement of sensation time]. Zeitschrift für Sinnesphysiologie, 54, 58-78.

GebHard, J. W., \& Mowbray, G. H. (1959). On discriminating the rate of visual flicker and auditory flutter. American Journal of Experimental Psychology, 72, 521-529.

Getzmann, S. (2007). The effect of brief auditory stimuli on visual apparent motion. Perception, 36, 1089-1103.

Getzmann, S., Lewald, J., \& Guski, R. (2004). Representational momentum in spatial hearing. Perception, 33, 591-599.

Grantham, D. W. (1986). Detection and discrimination of simulated motion of auditory targets in the horizontal plane. Journal of the Acoustical Society of America, 79, 1939-1949.

Halpern, A. R., \& Kelly, M. H. (1993). Memory biases in left versus right implied motion. Journal of Experimental Psychology: Learning, Memory, \& Cognition, 19, 471-484.

HANDEL, S. (1988). Space is to time as vision is to audition: Seductive but misleading. Journal of Experimental Psychology: Human Perception \& Performance, 14, 315-317.

HaRris, J. D., \& SERgEANT, R. L. (1971). Monaural-binaural minimum audible angles for a moving sound source. Journal of Speech \& Hearing Research, 14, 618-629.

Heron, J., Whitaker, D., \& McGraw, P. V. (2004). Sensory uncertainty governs the extent of audio-visual interaction. Vision Research, 44, 2875-2884

Hubbard, T. L. (1990). Cognitive representation of linear motion: Possible direction and gravity effects in judged displacement. Memory \& Cognition, 18, 299-309.

Hubbard, T. L. (1995a). Auditory representational momentum: Surface form, direction, and velocity effects. American Journal of Psychology, 108, $255-274$

HuBBARD, T. L. (1995b). Environmental invariants in the representation of motion: Implied dynamics and representational momentum, gravity, friction, and centripetal force. Psychonomic Bulletin \& Review, 2, 322-338.

HubBaRd, T. L. (1997). Target size and displacement along the axis of implied gravitational attraction: Effects of implied weight and evidence of representational gravity. Journal of Experimental Psychology: Learning, Memory, \& Cognition, 23, 1484-1493.

HubBaRD, T. L. (2005). Representational momentum and related displacements in spatial memory: A review of the findings. Psychonomic Bulletin \& Review, 12, 822-851.

Hubbard, T. L., \& Bharucha, J. J. (1988). Judged displacement in apparent vertical and horizontal motion. Perception \& Psychophysics, 44, 211-221.

Hubbard, T. L., \& Courtney, J. R. (2010). Cross-modal influences on representational momentum and representational gravity. Perception, 39, $851-862$.

JACK, C. E., \& Thurlow, W. R. (1973). Effects of degree of visual association and angle of displacement on the "ventriloquism" effect. Perceptual \& Motor Skills, 37, 967-979.

JoHnStON, H. M., \& JoNES, M. R. (2006). Higher order pattern structure influences auditory representational momentum. Journal of Experimental Psychology: Human Perception \& Performance, 32, 2-17.

Jordan, J. S., Stork, S., Knuf, L., Kerzel, D., \& Müsseler, J. (2002). Intentionality in perception/action space. In W. Prinz \& B. Hommel (Eds.), Attention and performance XIX: Common mechanisms in perception and action (pp. 158-176). Oxford: Oxford University Press.

Kelly, M. H., \& Freyd, J. J. (1987). Explorations of representational momentum. Cognitive Psychology, 19, 369-401.
KERZEL, D. (2000). Eye movements and visible persistence explain the mislocalization of the final position of a moving target. Vision Research, 40, 3703-3715.

Kerzel, D. (2006). Why eye movements and perceptual factors have to be controlled in studies on "representational momentum." Psychonomic Bulletin \& Review, 13, 166-173.

Kubovy, M. (1988). Should we resist the seductiveness of the space:time::vision:audition analogy? Journal of Experimental Psychology: Human Perception \& Performance, 14, 318-320.

McKee, S. P., \& Watamaniuk, S. N. J. (1994). The psychophysics of motion perception. In A. T. Smith \& R. J. Snowden (Eds.), Visual detection of motion (pp. 85-114). London: Academic Press.

Morein-Zamir, S., Soto-Faraco, S., \& Kingstone, A. (2003). Auditory capture of vision: Examining temporal ventriloquism. Cognitive Brain Research, 17, 154-163.

NAGAI, M., \& SAIKI, J. (2005). Illusory motion and representational momentum. Perception \& Psychophysics, 67, 855-866.

NAGAI, M., \& YAGI, A. (2001). The pointedness effect on representational momentum. Memory \& Cognition, 29, 91-99.

NiJHaWan, R. (1994). Motion extrapolation in catching. Nature, 370, 256-257.

PeLLI, D. G. (1997). The VideoToolbox software for visual psychophysics: Transforming numbers into movies. Spatial Vision, 10, 437-442.

Perrott, D. R., \& Musicant, A. D. (1977). Minimum auditory movement angle: Binaural localization of moving sound sources. Journal of the Acoustical Society of America, 62, 1463-1466.

RAdEAU, M. (1994). Auditory-visual spatial interaction and modularity. Current Psychology of Cognition, 13, 3-51.

RAdeAU, M., \& BerTelson, P. (1987). Auditory-visual interaction and the timing of inputs. Thomas (1941) revisited. Psychological Research, 49, 17-22.

ReEd, C. L., \& Vinson, N. G. (1996). Conceptual effects on representational momentum. Journal of Experimental Psychology: Human Perception \& Performance, 22, 839-850.

Sanabria, D., Soto-Faraco, S., Chan, J. S., \& Spence, C. (2004). When does visual perceptual grouping affect multisensory integration? Cognitive, Affective, \& Behavioral Neuroscience, 4, 218-229.

Sekuler, R., Sekuler, A. B., \& Lau, R. (1997). Sound alters visual motion perception. Nature, $\mathbf{3 8 5}, 308$.

Shams, L., Kamitani, Y., \& Shimojo, S. (2000). Illusions. What you see is what you hear. Nature, $\mathbf{4 0 8}, 788$.

Shams, L., Kamitani, Y., \& Shimojo, S. (2002). Visual illusion induced by sound. Cognitive Brain Research, 14, 147-152.

Shipley, T. (1964). Auditory flutter-driving of visual flicker. Science, $145,1328-1330$

Slutsky, D. A., \& Recanzone, G. H. (2001). Temporal and spatial dependency of the ventriloquism effect. NeuroReport, 12, 7-10.

Soto-Faraco, S., Kingstone, A., \& Spence, C. (2003). Multisensory contributions to the perception of motion. Neuropsychologia, 41, 1847-1862.

Soto-Faraco, S., Lyons, J., Gazzaniga, M., Spence, C., \& KingSTONE, A. (2002). The ventriloquist in motion: Illusory capture of dynamic information across sensory modalities. Cognitive Brain Research, 14, 139-146.

Soto-Faraco, S., Spence, C., \& Kingstone, A. (2004). Cross-modal dynamic capture: Congruency effects in the perception of motion across sensory modalities. Journal of Experimental Psychology: Human Perception \& Performance, 30, 330-345.

SPENCE, C. (2007). Audiovisual multisensory integration. Acoustical Science \& Technology, 28, 61-70.

Thornton, I. M. (2002). The onset repulsion effect. Spatial Vision, 15 , 219-243.

VInSON, N. G., \& REeD, C. L. (2002). Sources of object-specific effects in representational momentum. Visual Cognition, 9, 41-65.

VRoomen, J., \& DE Gelder, B. (2004). Temporal ventriloquism: Sound modulates the flash-lag effect. Journal of Experimental Psychology: Human Perception \& Performance, 30, 513-518.

Watanabe, K., \& Shimojo, S. (2001). When sound affects vision: Effects of auditory grouping on visual motion perception. Psychological Science, 12, 109-116.

WELCH, R. B. (1999). Meaning, attention, and the "unity assumption" in the intersensory bias of spatial and temporal perceptions. In G. Aschersleben, T. Bachmann, \& J. Müsseler (Eds.), Cognitive contributions 
to the perception of spatial and temporal events (pp. 371-387). Amsterdam: Elsevier.

Welch, R. B., DuttonHurt, L. D., \& Warren, D. H. (1986). Contributions of audition and vision to temporal rate perception. Perception \& Psychophysics, 39, 294-300.

WELCH, R. B., \& WARREN, D. H. (1980). Immediate perceptual response to intersensory discrepancy. Psychological Bulletin, 88, 638-667.

WELCH, R. B., \& WARREN, D. H. (1986). Intersensory interactions. In K. R. Boff, L. Kaufman, \& J. P. Thomas (Eds.), Handbook of perception and human performance: Sensory processes and perception (pp. 1-36). New York: Wiley.

\section{NOTES}

1. Some participants in the pilot experiment pointed out that they sometimes perceived motion between the visual target and the probe when the probe was presented on the trajectory of the visual target. This might prevent the participants from making precise judgments about the vanishing position. To avoid this confounding effect, the probe was presented above the trajectory of the visual motion. Whereas this manipulation was slightly different from those in the other RM studies, a reliable amount of RM was obtained.

2. To obtain subjective vanishing positions of a moving visual stimulus, the participants carried out a probe judgment task (left-right forced choice task) in the present study, and the probe position was determined by the staircase method. The staircase method has not been standard for measuring the size of visual RM but was adopted in the present study because many parameters had to be manipulated and the participants would have had to undergo too many trials to make precise judgments if a method of constant stimuli had been adopted. For exactly the same reason, Experiment 1 was divided into two blocks: the prior-offset block and the subsequent-offset block. The subsequent experiments (2-4) were also divided into two blocks in accordance with the procedure in Experiment 1.

3. In Experiment 1, 3 of 4 naive participants did not notice the differences in the timing of the visual and auditory offsets, whereas the other participant reported that, on some trials, the sound disappeared first, whereas on others, the visual target disappeared first. In the following experiments (Experiments 2-4), no naive participant noticed the difference in the timing of visual and auditory offsets. In our pilot experiment, a $159.6-\mathrm{msec}$ difference in the audiovisual offset was long enough to be noticed. One of the reasons why the 3 participants did not notice the difference in the audiovisual offset in Experiment 1 might be that they concentrated on the visual task with fine discriminations, and the sounds were assumed to be irrelevant to the task.

(Manuscript received September 4, 2009; revision accepted for publication June 8, 2010.) 